\title{
Reflectionless Grating Coupling for Silicon-on-Insulator Integrated Circuits
}

\author{
D. Vermeulen, Y. De Koninck, Y. Li, E. Lambert, W. Bogaerts, R. Baets, G. Roelkens \\ Photonics Research Group, Department of Information Technology, \\ Ghent University - imec, B-9000 Ghent, Belgium
}

\begin{abstract}
We propose a novel grating coupler design which is inherently reflectionless by focusing the reflected light away from the entrance waveguide. The grating coupler design is investigated by means of 3D FDTD simulations.
\end{abstract}

\section{Introduction}

Photonic integration is considered to be a key technology for future advancements in optical communication technologies. Scaling down the optical building blocks enables complex and ultra-compact photonic circuits at a fraction of the cost of conventional systems consisting out of discrete components. Choosing an appropriate platform for developing this miniaturization technology is guided by functionality, compatibility, performance, yield and cost. Regarding the last two issues, Silicon-on-Insulator (SOI) is by far the leading technology for low-cost and high-volume photonic integration since it can benefit from the processes developed in the mature electronics industry. Although the high refractive index contrast of SOI reduces the footprint of the integrated photonic devices considerably, it becomes more difficult to achieve a high-performance mode-size convertor between a single-mode optical fiber $\left(100 \mu \mathrm{m}^{2}\right)$ and an integrated optical waveguide $\left(0.1 \mu \mathrm{m}^{2}\right)$. A possible solution is a grating coupler which is a periodic structure that couples light out of the chip to free space or to an optical fiber [1]. Fiber-to-chip grating couplers with very high efficiency have been demonstrated [2]. However, as most papers focus on coupling efficiency, polarization dependency, ease of fabrication and bandwidth, a solution for the back reflection of these grating couplers is never investigated. A typical reflection back into the waveguide for a high-efficiency grating coupler is around $-17 \mathrm{~dB}$ [3]. Other reported high back reflections are $-10 \mathrm{~dB}[4],[5]$ and $-8 \mathrm{~dB}$ [6]. These amounts of back reflections are unacceptable for integrated circuits which contain integrated lasers. Furthermore, back reflections are highly unwanted in interferometer based designs.

\section{Theoretical Outline}

For grating couplers there are two main sources which contribute to the reflection back into the waveguide. The most important source is the second order reflection of the grating. Typically one eliminates this second order reflection by tilting the optical fiber under a small angle of around $10^{\circ}$ with respect to the surface normal [7]. The second source of back reflection is due the Fresnel reflection at the grating coupler interface. This reflection is highly dependent on the grating structure itself and is very difficult to eliminate. In the proposed grating coupler design, we will refocus these two sources of reflection away from the entrance waveguide thereby extinguishing the reflection almost completely. In [8] a compact focusing grating coupler is presented which uses a curved grating to focus the coupled light onto a single-mode waveguide, achieving an eight-fold length reduction as compared to a standard linear grating and adiabatic taper, without performance penalty. For the reflectionless grating coupler we follow the same design rules. The grating is curved such that a plain wavefront originating from the tilted optical fiber is phase-matched to the TE-polarized modes of a broad SOI waveguide, so that the wavefront is curved cylindrically and focusing occurs in the center of curvature of the wavefront. When the top surface of the waveguide is chosen to be the $(Y, Z)$ plane of a right handed Cartesian coordinate system, with $z$ along the waveguide axis and the origin chosen to be in the desired focal point we obtain grating lines that are confocal ellipses $E_{q_{0}}, E_{q_{0}+1}, \ldots, E_{q_{0}+n}$ with the common focal point $f_{1, q_{0}}=f_{1, q_{0}+1}=\ldots=$ $f_{1, q_{0}+n}$ at the origin (see Fig. 1a):

$$
\frac{\left(z-\frac{q \lambda_{0} n_{t} \sin \theta}{n_{e f f}^{2}-n_{t}^{2} \sin ^{2} \theta}\right)^{2}}{\left(\frac{q \lambda_{0} n_{e f f}}{n_{e f f}^{2}-n_{t}^{2} \sin ^{2} \theta}\right)^{2}}+\frac{y^{2}}{\left[\frac{q \lambda_{0}}{\left(n_{e f f}^{2}-n_{t}^{2} \sin ^{2} \theta\right)^{1 / 2}}\right]^{2}}=1
$$


where $q$ is an integer number for each grating line, $\theta$ is the angle between the fiber and the chip surface normal, $n_{t}$ is the refractive index of the environment, $\lambda_{0}$ is the vacuum wavelength and $n_{e f f}$ is the effective refractive index felt by the cylindrical wave in the slab and grating area. The position of the second focal points is given by

$$
f_{2, q}(z, y)=\left(\frac{2 q \lambda_{0} n_{t} \sin \theta}{n_{\text {eff }}^{2}-n_{t}^{2} \sin ^{2} \theta}, 0\right)
$$

The phase-matching condition is fulfilled for all parts of the confocal ellipses. We can thus choose which part of the ellipse we use for designing a grating coupler because every part of the ellipse will couple out the light under the same angle $\theta$. Normally one takes the right part of the confocal ellipses as shown in Fig. 1b, such that the propagation vector $\vec{\beta}$ of the entrance waveguide is parallel to the projected wave vector $\vec{k}_{i n, z}$ of the diffracted light (waveguide angle $\alpha=0^{\circ}$ ). Although this seems like the most logical choice, this design will introduce the largest back reflection since the reflection from the grating lines will be refocused to the second focal points and coupled back into the entrance waveguide. This is solved by using another part of the confocal ellipses as grating coupler and rotating the waveguide with an angle $\alpha$ of for example $45^{\circ}$, see Fig. 1c. In this design the focal points and the grating are not inline hence the grating reflection is focused away from the entrance waveguide into a slab region, thereby fully eliminating the back reflection.

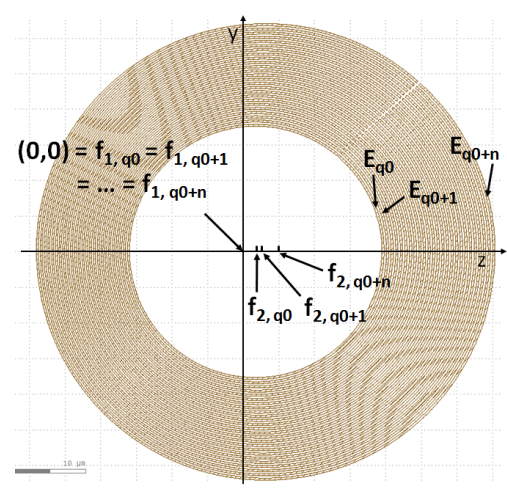

(a) Confocal ellipses (common focal point)

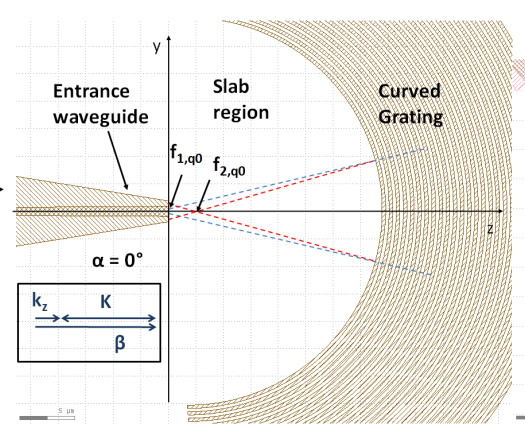

(b) $\alpha=0^{\circ}$

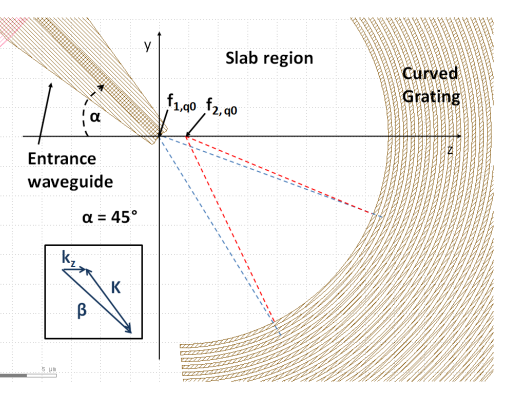

(c) $\alpha=45^{\circ}$

Fig. 1: Reflection from curved grating couplers for different waveguide angles $\alpha$. For $z=0$ the k-diagram is drawn $(\mathrm{K}=$ the local reciprocal lattice vector).

\section{Simulations}

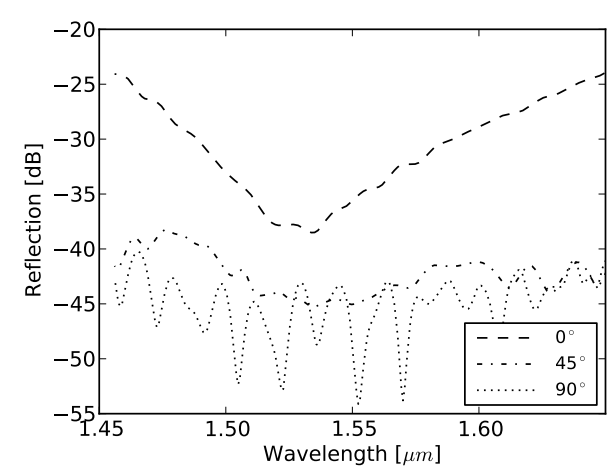

(a) Shallow etched grating couplers

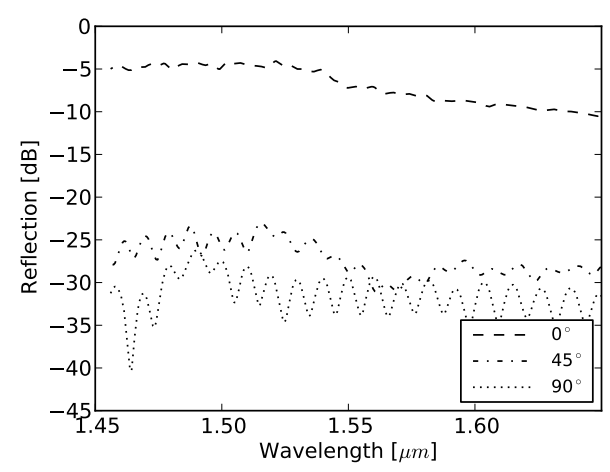

(b) Fully etched grating couplers

Fig. 2: Reflection spectra for shallow and fully etched grating couplers, each time for different values of the waveguide angle $\alpha$.

The grating couplers were simulated using full-vectorial 3D FDTD software [9]. To calculate the reflection spectra, a pulse with a Gaussian time profile was launched in the single-mode access waveguide and the reflected flux was 
measured in that same waveguide. Figure 2 shows the reflection spectra of the proposed grating coupler for different waveguide angles $\alpha$. For the shallow etched gratings ( $70 \mathrm{~nm}$ etch in a $220 \mathrm{~nm}$ Si core), the back reflection decreases around $10 \mathrm{~dB}$ to $15 \mathrm{~dB}$ thereby achieving typical back reflections of optical fiber connectors $(-40 \mathrm{~dB})$. For fully etched gratings $(220 \mathrm{~nm}$ Si core), the back reflection decreases from $-5 \mathrm{~dB}$ to $-25 \mathrm{~dB}$ thereby making these gratings useful for integrated circuits. The coupling efficiency was calculated by exciting a single frequency continuous wave in the access waveguide and calculating the overlap between the optical field above the coupler and the mode profile of a single-mode fiber $\left(\theta \approx 15^{\circ}\right)$, normalized to the power in the access waveguide. We found that the substrateless shallow etched design has a coupling efficiency of $33 \%$ independent of the waveguide angle $\alpha$.

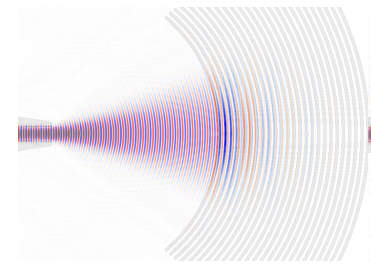

(a) $\alpha=0^{\circ}$

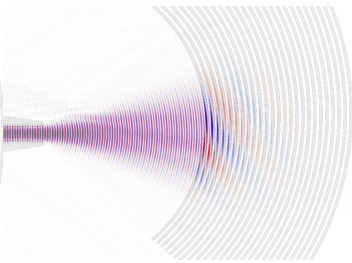

(b) $\alpha=45^{\circ}$

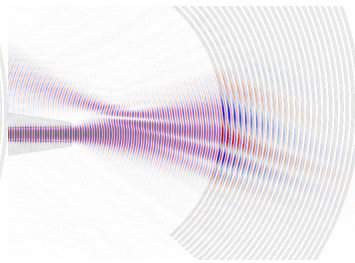

(c) $\alpha=90^{\circ}$

Fig. 3: Cross-sections of the electric field for a shallow etched grating coupler for different waveguide angles $\alpha$. The color scale is saturated to visualize the reflection

\section{Conclusion}

We have investigated a reflectionless grating coupler design by means of 3D FDTD simulations. The reflection could be decreased down to $-45 \mathrm{~dB}$, thereby realizing the same back reflection performance of a typical optical fiber connector, without introducing a coupling efficiency penalty. Furthermore, we showed that this method can be used to reduce the reflection of highly reflective $(-5 \mathrm{~dB})$ grating couplers to useful reflections of $-25 \mathrm{~dB}$. These devices could become key components in interferometer based designs and circuits using integrated lasers.

\section{Acknowledgements}

Diedrik Vermeulen thanks the Institute for the Promotion of Innovation by Science and Technology in Flanders (IWT) for a grant. Yannick De Koninck acknowledges the Research Foundation - Flanders (FWO) for a research grant.

\section{References and links}

1. D. Taillaert, W. Bogaerts, P. Bienstman, T. Krauss, P. Van Daele, I. Moerman, S. Verstuyft, K. De Mesel, and R. Baets, "An out-of-plane grating coupler for efficient butt-coupling between compact planar waveguides and single-mode fibers," IEEE Journal of Quantum Electronics, vol. 38, no. 7, pp. 949-955, Jul. 2002. [Online]. Available: http://ieeexplore.ieee.org/lpdocs/epic03/wrapper.htm?arnumber=1017613

2. D. Vermeulen, S. Selvaraja, P. Verheyen, G. Lepage, W. Bogaerts, P. Absil, D. Van Thourhout, and G. Roelkens, "High-efficiency fiber-to-chip grating couplers realized using an advanced CMOS-compatible silicon-on-insulator platform." Optics express, vol. 18, no. 17, pp. 18 278-83, Aug. 2010. [Online]. Available: http://www.ncbi.nlm.nih.gov/pubmed/20721220

3. A. Mekis, S. Gloeckner, G. Masini, A. Narasimha, T. Pinguet, S. Sahni, and P. Dobbelaere, "A Grating-Coupler-Enabled CMOS Photonics Platform," Selected Topics in Quantum Electronics, IEEE Journal of, no. 99, pp. 1-12, 2010. [Online]. Available: http://ieeexplore.ieee.org/xpls/abs_all.jsp?arnumber $=5645652$

4. L. Zhou, Z. Li, Y. Zhu, Y. Li, Z. Fan, W. Han, Y. Yu, and J. Yu, "A novel highly efficient grating coupler with large filling factor used for optoelectronic integration," Chinese Physics B, vol. 19, p. 124214, 2010. [Online]. Available: http://iopscience.iop.org/1674-1056/19/12/124214

5. X. Chen and H. Tsang, "Polarization-Independent Grating Couplers for Silicon-on-Insulator Nanophotonic Waveguides," OPTICS LETTERS, vol. 36, no. 6, pp. 796-798, 2011. [Online]. Available: http://www.opticsinfobase.org/ol/upcoming_pdf.cfm?id=139464

6. H. Tsang, "Nanoholes Grating Couplers for Coupling Between Silicon-on-Insulator Waveguides and Optical Fibers," IEEE Photonics Journal, vol. 1, no. 3, pp. 184-190, Sep. 2009. [Online]. Available: http://ieeexplore.ieee.org/lpdocs/epic03/wrapper.htm?arnumber=5232876

7. D. Taillaert, P. Bienstman, and R. Baets, "Compact efficient broadband grating coupler for silicon-on-insulator waveguides." Optics letters, vol. 29, no. 23, pp. 2749-51, Dec. 2004. [Online]. Available: http://www.ncbi.nlm.nih.gov/pubmed/15605493

8. F. Van Laere, T. Claes, J. Schrauwen, S. Scheerlinck, W. Bogaerts, D. Taillaert, L. O'Faolain, D. Van Thourhout, and R. Baets, "Compact Focusing Grating Couplers for Silicon-on-Insulator Integrated Circuits," IEEE Photonics Technology Letters, vol. 19, no. 23, pp. 1919-1921, Dec. 2007. [Online]. Available: http://ieeexplore.ieee.org/lpdocs/epic03/wrapper.htm?arnumber=4383198

9. A. F. Oskooi, D. Roundy, M. Ibanescu, P. Bermel, J. Joannopoulos, and S. G. Johnson, "Meep: A flexible free-software package for electromagnetic simulations by the FDTD method," Computer Physics Communications, vol. 181, no. 3, pp. 687-702, Mar. 2010. [Online]. Available: http://linkinghub.elsevier.com/retrieve/pii/S001046550900383X 